EPJ Web of Conferences 59, 03016 (2013)

DOI: $10.1051 /$ epjconf/20135903016

(C) Owned by the authors, published by EDP Sciences, 2013

\title{
Polarization: A must for fusion
}

\author{
J.-P. Didelez ${ }^{1, a}$ and C. Deutsch ${ }^{2}$
}

${ }^{1}$ Institut de Physique Nucléaire, CNRSIIN2P3 \& Université Paris-Sud (UMR-CNRS 8608) Bât. 100, 91406 Orsay Cedex, France

${ }^{2}$ Laboratoire de Physique des Gaz et des Plasmas, Université Paris-Sud (UMR-CNRS 8578) Bât. 210, 91405 Cedex, France

\begin{abstract}
The complete polarization of DT fuel would increase the fusion reactivity by $50 \%$ in magnetic as well as in inertial confinements. The persistence of polarization in a fusion process could be tested, using a terawatt laser hitting a polarized $H D$ target. The polarized deuterons heated in the plasma induced by the laser can fuse producing a ${ }^{3} \mathrm{He}$ and a neutron in the final state. The angular distribution of the emitted neutrons and the change in the corresponding total Cross Section (CS) can sign the polarization persistence. The polarization of solid $\mathrm{H}_{2}, \mathrm{D}_{2}$ or $T_{2}$ Hydrogen isotopes is very difficult. However, it has been possible to polarize $H D$, a hetero-molecular form of Hydrogen, by static polarization, at very low temperature and very high field. The radioactivity of $D T$ molecules forbids there high polarization by the static method, therefore one has to develop the Dynamic Nuclear Polarization (DNP) by RF transitions. The DNP of HD has been investigated in the past. The magnetic properties of $H D$ and $D T$ molecules are very similar, it is therefore expected that any polarization result obtained with $H D$ could be extrapolated to $D T$.
\end{abstract}

\section{INTRODUCTION}

The polarization of $D$ and $T$ nuclei should increase their reactivity when used as fuel material in fusion processes induced either by magnetic or by inertial confinement. The fusion reaction:

$$
D+T \rightarrow \alpha+\text { neutron }+17.6 \mathrm{MeV}
$$

goes mainly through the excitation of an ${ }^{5} \mathrm{He} 3 / 2^{+}$intermediate state, resulting from the coupling of the spins 1 and $1 / 2$ of the $D$ and $T$ nuclei to a total spin $S=3 / 2$. Without polarization of $D$ and $T$, the statistical distribution of the six possible states gives four $S=3 / 2$ and two $S=1 / 2$ states. Only the $3 / 2$ states can produce the intermediate $3 / 2$ resonance. With $100 \%$ parallel polarization of $D$ and $T$, all states would contribute to the fusion, increasing the reactivity by $50 \%$. In addition, the polarization determines the direction in which the reaction products are emitted, the neutron having a $\sin ^{2} \theta$ distribution. This can be very useful to reduce damage or activation of costly equipments. The question is to know if the polarization will persist in dense and hot plasmas, as suggested by Kulsrud et al. for magnetic [1] and More [2] for inertial confinements.

\section{METHOD}

We propose to investigate the polarization persistence using the reactions:

$$
\mathrm{H}+\mathrm{D} \rightarrow{ }^{3} \mathrm{He}+\gamma+5.5 \mathrm{MeV}
$$

\footnotetext{
ae-mail: didelez@ipno.in2p3.fr
}

This is an Open Access article distributed under the terms of the Creative Commons Attribution License 2.0, which permits unrestricted use, distribution, and reproduction in any medium, provided the original work is properly cited. 


$$
D+D \rightarrow{ }^{3} \mathrm{He}+n+3.3 \mathrm{MeV}
$$

induced by fusion of polarized protons and deuterons heated in a plasma. It is anticipated that the angular distributions of final state products as well as significant changes in the fusion rates can be measured and related to the persistence of the polarization [3].

\section{TENTATIVE SETUP}

At IPN Orsay, we have developed the static polarization of $H D$ molecules for samples as large as $25 \mathrm{~cm}^{3}$. It has been demonstrated that the distillation and the ageing technique allow getting relaxation times larger than one week, even at $1.5 \mathrm{~K}$ and $1.0 \mathrm{~T}$. Proton polarization in excess of $60 \%$ and deuteron vector polarization higher than $14 \%$ have been achieved [4].

It is advocated that a terawatt laser hitting a piece of polarized $H D$ will induce locally a plasma hot enough to allow the fusion reactions (2) and (3) to take place and to be measured. If both $H$ and $D$, namely the proton and the deuteron of the $H D$ molecules are polarized in the same direction and have kept their polarization in the fusion process, the $\gamma$ ray or the neutron will be emitted with some angular distribution relative to the polarization axis. Also, the fusion rate will depend drastically on the initial states polarizations. Back in 1970, a French group of the CEA reported the observation of neutron emission from $D D$ fusion after focusing a $3 \mathrm{GW}$ fast laser on a piece of $D_{2}$ ice [5]. More recently, quantitative data resulting from the irradiation of $C_{2} D_{4}$ targets with laser pulses $(200 \mathrm{~mJ}, 160 \mathrm{fs}, 4.5 \mu \mathrm{m}$ FWHM, $790 \mathrm{~nm}, \sim 10^{18} \mathrm{~W} / \mathrm{cm}^{2}, 10 \mathrm{~Hz}$ ) have been published. A total rate of 140 neutrons/shot could be produced through the fusion reaction (3) [6]. It should be mentioned that with $200 \mathrm{~mJ} / \mathrm{shot}$, the laser repetition rate can be adjusted to prevent melting of the target. Without cooling power provided by the holding cryostat, 1,000 such laser shots would be necessary to melt completely $25 \mathrm{~cm}^{3}$ of solid $H D$.

\section{THE « FEW-BODY» PROBLEMS}

For the radiative capture (2), the experiment is essentially based on the angular distribution of the radiated $\gamma$ ray with respect to the polarization axis. Assuming that $H$ and $D$ nuclei collide from all directions in a hot plasma, with a total spin $S=3 / 2$ (quartet transitions: $\sigma_{4}$, namely $100 \%$ polarization), while an unpolarized plasma involves also transitions from total spin $S=1 / 2$ (doublet transitions: $\sigma_{2}$ ), the angular dependence has the form:

$$
d \sigma_{4} / d \omega \sim\left(1+\cos ^{2} \theta\right) .
$$

At the low energies considered here, other contributions containing isotropic and $\left(3 \cos ^{2} \theta-1\right)$ terms can be neglected [7]. This means that the quartet transitions contribute mainly at forward angles, namely in the direction of the laser beam. It is out of question to put a $\gamma$ ray detector in this region of high electron background. A $10^{18} \mathrm{~W} / \mathrm{cm}^{2}$ laser pulse can produce electrons and $\gamma$ rays with energies as high as $10 \mathrm{MeV}$ with intensities several order of magnitude larger than the few counts due to the radiative capture. The corresponding signal would completely obscure the radiative $\gamma$ rays. This makes the radiative capture reaction fairly difficult to exploit and even hopeless from an experimental point of view.

The hadronic fusion reaction (3), producing 2.45 MeV neutrons which are much easier to detect in a high background than $\gamma$ rays, offers an alternative possibility. It has been argued that the Cross Section (CS) should be significantly reduced if the interacting deuterons have parallel vector polarizations (i.e., with total spin $S=2$, namely quintet transitions $\sigma_{5}$ ). The large Quintet Suppression Factor (QSF) is confirmed by recent calculations, with QSF going from 0.5 at $100 \mathrm{keV}$ to 0.2 at $4 \mathrm{MeV}$ [8]. Moreover, the total CS is in the range of $100 \mathrm{mb}$, to be compared to $100 \mu \mathrm{b}$ for the electromagnetic reaction (2). In addition, the neutrons produced by quintet transitions are preferentially emitted in a direction perpendicular to the polarization axis, with a $\sin ^{2} \theta$ angular distribution [9]. In view of those considerations, the reaction $\mathrm{D}+\mathrm{D} \rightarrow{ }^{3} \mathrm{He}+n$ is the way to go. It should be noted that for a polarized 


\section{Dynamic Polarization of HD or DT}

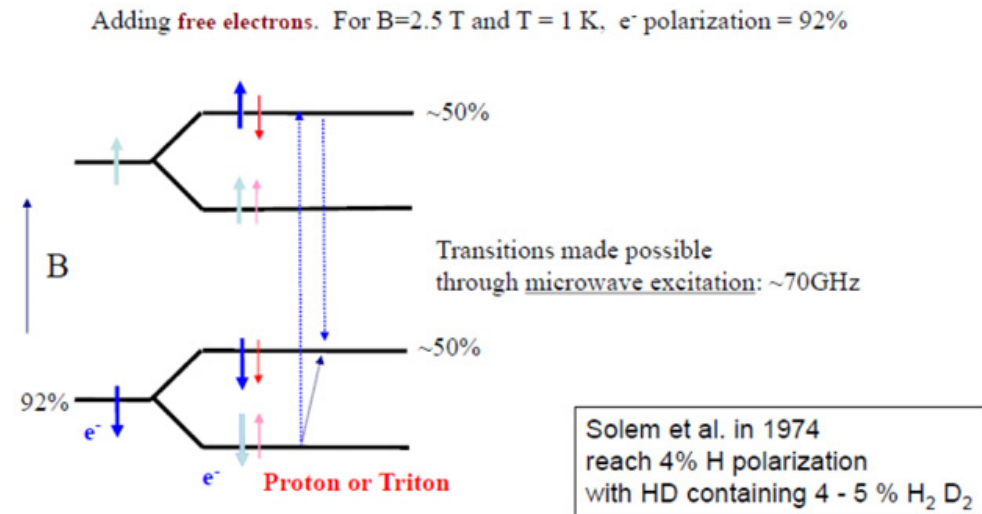

Initial concentration Needed

o- $\mathrm{H} 2:<0.02 \%$

p-D2: $<0.1 \%$

Proton relaxation time $\gg$ electron

Figure 1. Scheme of the DNP polarization of Protons or Tritons.

$H D$ target, it is possible to increase the $D$ polarization above $50 \%$ at the expense of the $H$ one, by transfer of the polarization of $H$ to $D$ by adiabatic fast passage [10-12]. Detailed description and discussion of the present experiment can be found in [3].

A corresponding proposal has been made at the Institute for Laser Engineering (ILE) in Osaka, Japan and has been accepted as the POLAF project (Polarization in Laser Fusion) [12]. The experiment is going on using the ILE equipment, in particular the MANDALA neutron multi-detector.

\section{POLARIZATION OF HYDROGEN ISOTOPES}

The full polarization of the DT fuel would increase the fusion reaction CS by $50 \%$. In the case of ICF, recent realistic simulations show that the required hot-spot temperature $(\mathrm{T})$ and areal density $(\rho \mathrm{R})$ can be reduced by about $15 \%$ for fully polarized nuclear fuel. Moreover, numerical simulations of a directly driven capsule show that the required laser power and energy to achieve high gain are significantly reduced, while the maximum achievable energy gain scales roughly as the fusion cross section [13]. These results refer only to a specific capsule and laser pulse configuration. Nevertheless, the trend should be valid in general cases and emphasizes the importance of the fuel polarization. Assuming that the persistence of the polarization in a fusion process has been demonstrated, there remains the problem of the polarization of the $D T$ fuel in inertial fusion reactors [14]. The polarization of solid $H_{2}, D_{2}$ or $T_{2}$ Hydrogen isotopes in their homo-molecular form appears to be very difficult, but not impossible.

It has been possible to polarize $H D$, an hetero-molecular form of Hydrogen, by static polarization, namely at very low temperature $(10 \mathrm{mK})$ and very high field $(17 \mathrm{~T})$ and to obtain after polarization, long relaxation times (more than a week) at higher temperatures (4 K) and lower fields (1 T) [4]. High static polarization of $D T$ would not work, because of the heat generated by the Tritium radioactivity ( $1 \mathrm{~W} / \mathrm{mol})$. Therefore, one has to count on the Dynamic Nuclear Polarization (DNP) using RF transitions. The magnetic properties of $H D$ and $D T$ molecules are very similar. It is therefore expected that any polarization result obtained with $H D$ could be extrapolated to $D T$, to the exception that the $D T$ radioactivity must be taken into account [15]. Although quite possible in principle, the DNP of $H D$ has been investigated in the past with moderate success [16]. Figure 1 shows the standard scheme 
of DNP polarization of $H D$ (see [14] for more detail). To be efficient one needs long relaxation times $T_{1}^{H}$ and $T_{1}^{D}$ for the nuclei and short relaxation times $T_{1}^{e}$ for the electrons. This is normally the case for distilled $H D$ [4]. Therefore, Solem's experiment should be repeated in order to define a protocol for optimum DNP polarization of $H D$ molecules that could possibly be applied to $D T$ molecules. It should be mentioned that the DNP polarization of $D_{2}$ and $H_{2}$ (or $T_{2}$ ) could also be investigated. The "ortho" configurations of those molecules represent a significant fraction of the statistical distributions and are in metastable states having long decay constants compared to a possible fast DNP process. The corresponding polarizations would be limited to $75 \%$ for $H_{2}$ (or $T_{2}$ ) and $83 \%$ for $D_{2}$ which is not so bad. Hopefully, the time necessary to bring a corresponding $D T$ target in the implosion region, from the cryostat to the center of the experimental chamber, could be made short enough compared to the relaxation times of the polarized nuclei. Pneumatic expulsion of cryogenic bullets has been used successfully in nuclear physics experiments reducing the transit time to a few ms. Such short times could be compatible with temperatures of the target closer to the triple point as usually required for target uniformity. One should also point out that $D T$ hetero-molecules could stand lower temperatures than a mixture of two different isotopes: $D_{2}$ and $T_{2}$, keeping nevertheless some uniformity.

\section{CONCLUSIONS}

A considerable effort is under way to produce energy using controlled fusion either by magnetic or by inertial confinement. Polarized fusion fuel is of great interest, both to increase the fuel reactivity and to control the direction in which reaction products are emitted. The question to know if the polarization will persist in a fusion process can be answered using existing experimental equipments and neutron detection. A signal of $10-20 \%$ on the neutron yield due to the persistence of the polarization should be « easily » measurable. The corresponding investigation is underway at ILE (Osaka). The polarization of the $D T$ fuel is a huge problem, but the impact of the polarization on fusion is so important that a revival of the field seems justified.

\section{References}

[1] R.M. Kulsrud et al., Phys. Rev. Lett. 49, 1248 (1982)

[2] R.M. More, Phys. Rev. Lett. 51, 396 (1983)

[3] J.-P. Didelez and C. Deutsch, Laser and Particle Beams 29, 169 (2011)

[4] S. Bouchigny et al., Nucl. Instr. and Meth. A 607, 271 (2009)

[5] F. Floux et al., Phys. Rev. A 1, 821 (1970)

[6] G. Pretzler et al., Phys. Rev. E 58, 1165534 (1998)

[7] M. Viviani et al., Phys. Rev. C 54, (1996); ibid. C 61, 064001 (2000) and private communication

[8] A. Deltuva and A. C. Fonseca, Phys. Rev. C 76, 021001(R) (2007); A. Deltuva et al., Phys. Lett. B 660, 471 (2008) and private communication

[9] H. Paetz gen. Schieck, Eur. Phys. J. A 44, 321 (2010)

[10] J.-P. Didelez, Nucl. Phys. News 4, 10 (1994)

[11] A. Honig et al., Nucl. Instr. and Meth. A 356, 39 (1995)

[12] 2011 Application Form for Joint Research, RCNP-ILE-IPN-LPGP Collaboration, Osaka, Japan

[13] M. Temporal et al., Nucl. Fusion 52, 103011 (2012)

[14] S. Bouchigny and J.-P. Didelez, Nucl. Instr. and Meth. A 577, 320 (2007)

[15] P. C. Souers et al., J. Vac. Technol. A 11(5), 2827 (1993)

[16] J.C. Solem, Nucl. Instr. and Meth. 117, 477 (1974) 